\title{
Optimization of Energy Consumption by Fuzzy Based Routing In Wireless Sensor Network
}

\author{
B. Meenakshi ${ }^{1}$, P. Anandhakumar ${ }^{2}$ \\ ${ }^{1}$ (Research Scholar, Department of Information Technology, MIT Campus Anna University, Chennai-44, India) \\ ${ }^{2}$ (Professor, Department of Information Technology, MIT Campus Anna University, Chennai-44, India)
}

\begin{abstract}
Maintaining the energy of sensors in Wireless Sensor Network (WSN) is important in critical applications. WSN consists of sensor nodes which sense the physical parameters such as temperature, humidity, pressure and light etc and send them to a fusion center namely Base Station (BS) from where one can get the value of physical parameters at any time. Man cannot often recharge the batteries which supplies the sensing device, transceiver and memory unit in the sensor node. So the usage of the battery power must be judicious in WSN. Earlier attempts have been made to prolong the network lifetime, but still it is a challenging task. In this paper we optimize the energy consumption of WSN by clustering and fuzzy based next hop selection.
\end{abstract}

Keywords: WSN; Base Station; Network Lifetime; clustering; next hop;

\section{INTRODUCTION}

The lifetime of a sensor network can be defined as the duration from the deployment of the network to the time when the first or the last sensor node runs out of energy. One of an energy-efficient technique to extend the lifetime of a sensor network is clustering. To extend the sensor lifetime, it is often coupled with the data aggregation. Each cluster selects one node as the cluster head. The data gathered from the sensors are forwarded to the cluster head first, and then to the sink. The cluster heads can aggregate the data from the sensors to minimize the amount of the data to be forwarded to the sink. Clusters can be organized hierarchically when the network size increases. This work is the extension of our previous [1], A Novel Energy efficient Surface water Wireless Sensor Network Algorithm (NES-WSN).

Sensor node is to sense the information; the sensed information is conveyed to BS through transceivers. From BS information can be sent to satellite. Like this arrangement so many BSs can be linked through internet. In definite time intervals the nodes which act as $\mathrm{CH}$ (Cluster Head), collects the information from their CMs (Cluster Members), aggregate the data and send them to BS. In NES-WSN algorithm the total area is divided into three regions based on the Time Factor (TF). Two CHs are selected. One is to collect information from CMs and other to forward the data to BS. In NES-WSN Algorithm the routing of data to BS is not clearly defined. In this work the next hop node is selected based on fuzzy rule based technique.

\section{RELATED WORK}

Wendi Rabiner Heinzelman et al. compared direct communication, Minimum Transmission Energy (MTE) method with his Low Energy Adaptive Clustering Hierarchy (LEACH)[2] In direct communication nodes can send information directly to the Base Station (BS). In case of direct communication, if BS is far away from node, the life of the battery reduced sooner. In MTE method, the node which has minimum transmission energy to transfer data is chosen to receive data from all other nodes and transfer data to BS. Here the node nearer to BS dies out quickly. Commonly used WSN architecture is by grouping nodes into clusters and the selected Cluster Head $(\mathrm{CH})$ communicates with BS. Changing the $\mathrm{CH}$ periodically is a must so as to reduce the stress on the same node since $\mathrm{CH}$ is communicating with all other nodes constantly and also with BS. LEACH is implemented in two phases. (i) Set up phase. (ii) Steady state phase. In set up phase the clusters are framed and for each cluster, $\mathrm{CH}$ is selected. LEACH uses coordination so as to enable scalability and robustness for dynamic networks, and combine data fusion into routing protocol to reduce amount of information transferred to BS. LEACH saves energy 8 times than MTE routing and direct transmission.

M. J. Handy et al. in his L-DCHS (LEACH with Deterministic CH selection) [3] increased the lifetime of a network. The threshold value is modified by including the remaining energy level available in each node.

Linping Wang et al. improved the L-DCHS algorithm in IEAL algorithm (Improved Energy-efficient Algorithm based on L-DCHS) [4] by making the nodes to know about the location of other nodes, secondly by appointing two CHs. The $\mathrm{MCH}$ (Master $\mathrm{CH}$ ) is elected by calculating the threshold value. The node factor is sent to $\mathrm{MCH}$ by all the nodes belong to the same cluster. The $\mathrm{MCH}$ select the $\mathrm{VCH}$ (Vice $\mathrm{CH}$ ) which is having highest node factor. The cluster maintenance is done by watching the current energy of the $\mathrm{MCH}$ and $\mathrm{VCH}$. If the current energy of $\mathrm{MCH}$ is less than 2 times the energy threshold value then the new cluster is formed with new CHs. It 
Optimization Of Energy Consumption By Fuzzy Based Routing In Wireless Sensor Network

is observed that in IEAL the lifetime improvement of WSN is 19.8\% for HND (Half Node Dies) and 6.7\% for LND (Last Node Dies).

Further the energy improvement is done in NEAW (Novel Energy-efficient Algorithm for WSN) [5] by splitting the total area into 3 regions. The 3 regions have different $\mathrm{MCH}$ and $\mathrm{VCH}$ whose functions are heterogeneous. The far VCH nodes send data to BS through multi hop. $\mathrm{MCH}$ receives data from all nodes and send them to $\mathrm{VCH}$. VCH is responsible for compressing the data and send to BS. The NEAW has $8.6 \%$ delays at time when the first node die, $24.2 \%$ improvement when there are half of the nodes alive, in comparison to L-DCHS. Moreover, NEAW can achieve even a $14.3 \%$ increase in LND.

WSN used for many real time applications. Many projects are going on throughout the world which uses the WSN to collect much more useful data. WINSOC project, Idukki, Kerala state, India[6] to detect Landslide, SmartDetect, IISC, Bangalore, India [7],[8],[9] for intruder detection purpose, ComonSense Net(CSN) project, Karnataka, India[10] for agriculture Management, MpWiNodeZ, Douro[11] for Precision viticulture of Grape Field.

\section{Fuzzy Based Routing}

The present work demonstrates how the next hop is selected using fuzzy based routing. In this method the next hop is selected using three parameters- Time Factor, Remaining Energy and distance.

\subsection{Estimation Metrics}

Time Factor (TF)

The BS sent the broadcast information to the node at time $\left(\mathrm{t}_{1}\right)$, the acknowledgement received from the node at time $\left(\mathrm{t}_{2}\right)$, then the TF $(\mathrm{t})$ is calculated using (1).

\section{Threshold value $\mathbf{T} \_\mathbf{N}$}

$$
\mathrm{TF}=\left(\mathrm{t}_{2}-\mathrm{t}_{1}\right)
$$

$T_{N(i)}=\frac{P}{1-P\left(\operatorname{rmod}\left(\frac{1}{P}\right)\right)}\left[\frac{E_{\text {rem }}(i)}{E_{\max }(i)}+\left(r_{n_{c h}} \operatorname{div}\left(\frac{1}{P}\right)\right)\left(1-\frac{E_{r e m}(i)}{E_{\max }(i)}\right)\right] \rightarrow(2)$

$\mathrm{P}->$ percentage of $\mathrm{CH}$ among nodes ' $\mathrm{n}$ ';

$\mathrm{i}->$ represents for any $\mathrm{i}^{\text {th }}$ node;

$\mathrm{r}->$ round no.;

$\mathrm{r}_{\mathrm{n} \_\mathrm{ch}} \mathrm{C}^{-}>$no. of rounds for which the node is not being $\mathrm{CH}$;

Threshold value includes remaining energy of $i_{\text {th }}$ node and maximum energy of the node. Threshold value calculated for the nodes which are not selected as PMN for the last (1/P) rounds by using equation (2), otherwise its value taken as zero. BS compares the random number with $\mathrm{T}_{-} \mathrm{N}$ and selects $\mathrm{P}$ number of PMNs whose random number generated is less than the T_N.[3],[4],[5]

Node Factor NF(i)

$$
N F(i)=\frac{E_{r e m}(i)}{T F(i)} \rightarrow(3)
$$

$\mathrm{NF}(\mathrm{i})$ is the node factor of $i^{\text {th }}$ node. $E_{\text {rem }}(i)$ is the remaining energy in $i^{\text {th }}$ node. TF(i) is the Time Factor of $i^{\text {th }}$ node. NF is high for nodes having more remaining energy and for nodes taking less time to communicate with BS.

\section{Transmission, receiving and remaining energy}

$$
\begin{gathered}
E_{\text {trans }}(k, d)=E_{\text {trans }_{\text {elec }}}(k)+E_{\text {trans amp }_{\text {amp }}}(k, d)=K E_{\text {elec }}+k \varepsilon_{\text {amp }} d^{2} \rightarrow(4) \\
E_{\text {recv }}(k)=E_{\text {recv } v_{\text {elec }}}(k)=k E_{\text {elec }} \rightarrow(5)
\end{gathered}
$$

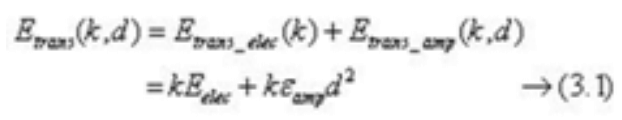

Where $\mathrm{E}_{\text {trans }}(\mathrm{k}, \mathrm{d})$ is the energy required to transmit $\mathrm{k}$ bits over distance d. Here the distance decided by the Time Factor (TF). TF is the summation of time duration to send the message from BS to node $\left(t_{1}\right)$ and got reply from node to BS $\left(t_{2}\right)$. The distance $d$ is calculated using (6)

$$
d=\left(t_{2}-t_{1}\right) * V \rightarrow(6)
$$

Where $\mathrm{E}_{\text {trans_elec }}(\mathrm{k})$ is the electrical energy required for transmitting $\mathrm{k}$ bits which is equal to $\mathrm{kE}_{\text {elec }}$. The energy spent for amplifying $\mathrm{k}$ bits to transmit over $\mathrm{d}$ distance is $\mathrm{E}_{\text {trans_amp }}(\mathrm{k}, \mathrm{d})$ and is equal to $\mathrm{k} \varepsilon_{\text {amp }} \mathrm{d}^{2}$. Energy 
Optimization Of Energy Consumption By Fuzzy Based Routing In Wireless Sensor Network required to receive $\mathrm{k}$ bits is $\mathrm{E}_{\text {recv }}(\mathrm{k})$ and is equal to $\mathrm{kE}_{\text {elec }}$. Velocity of radio signals is $\mathrm{V}$ and is equal to $300 \mathrm{~km} / \mathrm{sec}$

$$
E_{\text {rem }}=E_{\text {init }}-\left(E_{\text {trans }}+E_{\text {recv }}\right) \rightarrow(7)
$$

\subsection{NES-WSN algorithm}

TABLE I shows our previous work NES-WSN Algorithm. The NES-WSN Algorithm includes the following phases of works

$>$ Nodes Deployment, Cluster Formation, $\mathrm{CH}$ selection

$>$ Routing

Energy Maintenance

TABLE I : NES-WSN algorithm

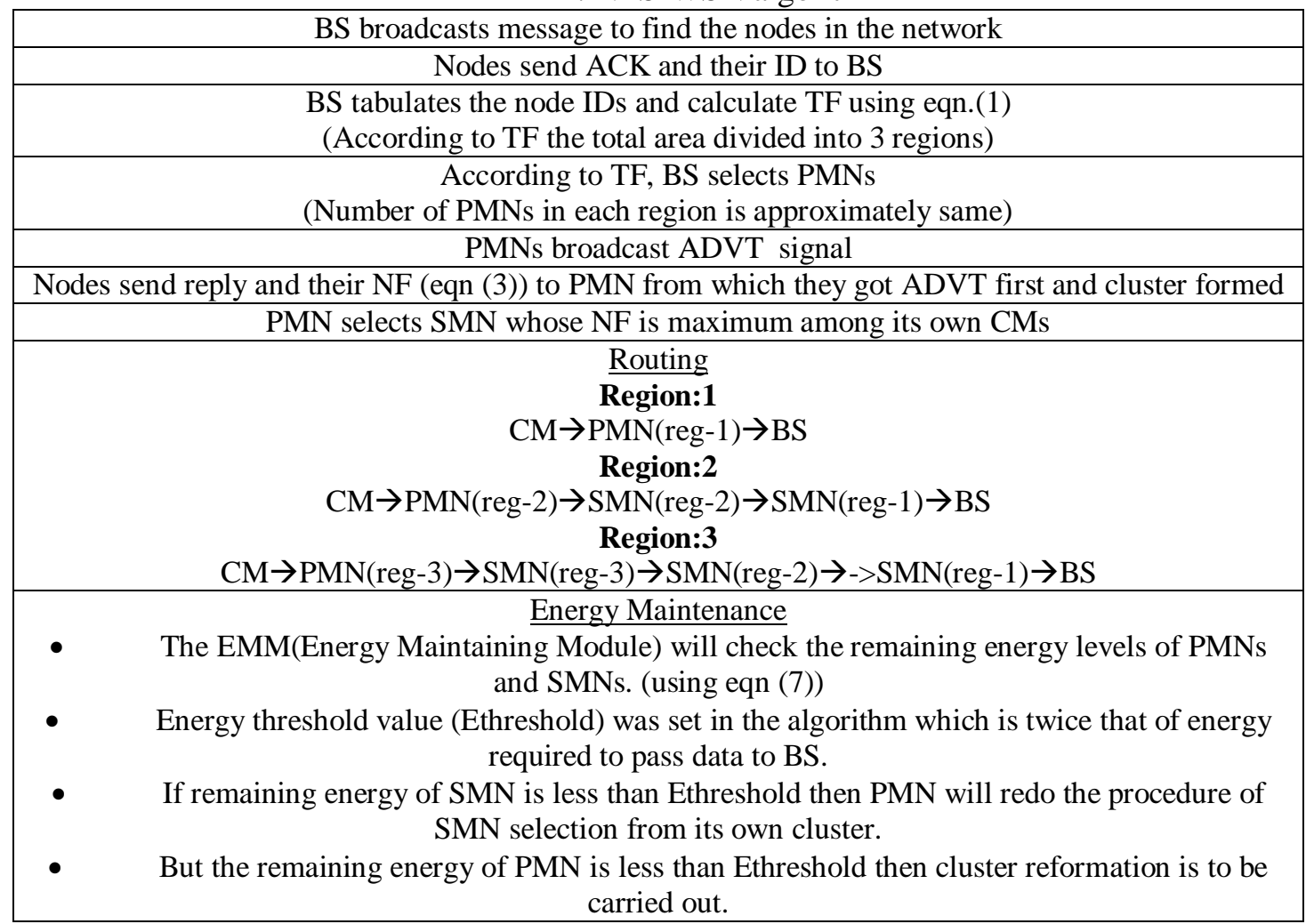

PMN $\rightarrow$ Primary Master Node; SMN $\rightarrow$ Secondary Master Node; $\mathrm{CM} \rightarrow$ cluster Member; ACK $\rightarrow$ Acknowledgement; ADVT $\rightarrow$ advertisement ; reg $\rightarrow$ Region; SMN and PMN are two CHs for a cluster.

\subsection{Proposed work}

In fuzzy based routing the data from CM pass on to the SMN of corresponding region. From SMN the data routed to the SMN of next region towards BS. In the next region there were so many SMNs. The data from ith node passed on to the jth node.

The Next hop selection Factor $\mathrm{NH}_{\mathrm{ij}}$ is given by eqn (8)

$$
N H_{i j}=a \cdot \frac{E_{r e m \_j}}{T F_{j}}+b \cdot \frac{1}{D_{i j}} \rightarrow(8)
$$

Where $a$ and $b$ are constants. $E_{r e m} j$ is remaining energy of jth node, $\mathrm{TF}_{\mathrm{j}}$ is the time factor of jth node and $D_{i j}$ is the distance between the nodes $i$ and $j$. The remaining energy, Time Factor and distance were given as inputs to the Fuzzy Inference System (FIS) (Fig 1) and by using 8 rules (listed in TABLE II) in the inference engine the probability of being next hop node was calculated. Fig 2 shows the rules used to get the output and Fig 3 and Fig 4 shows the output. 


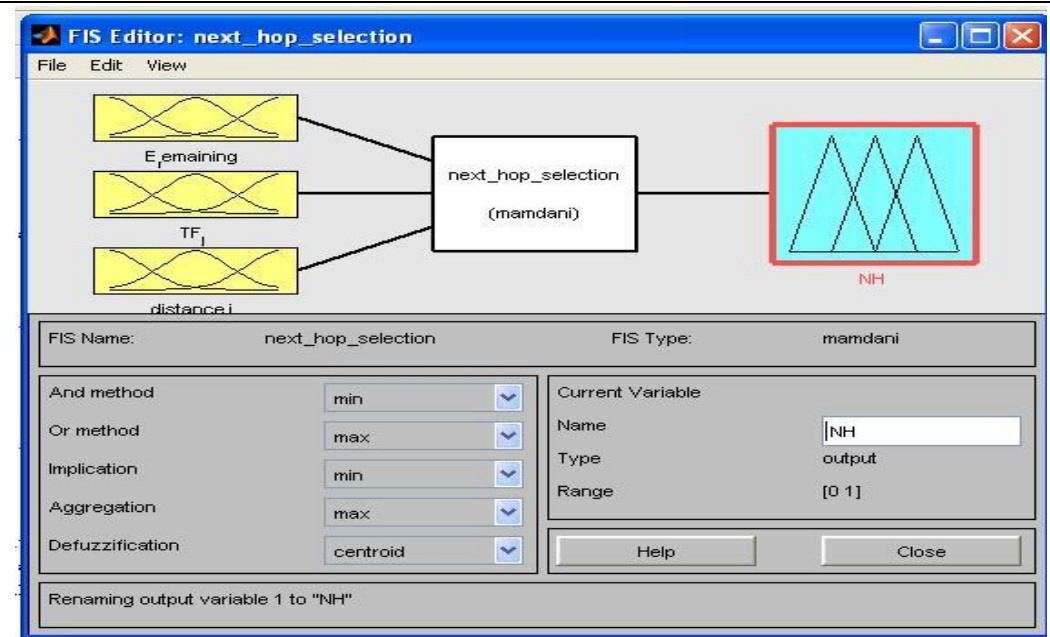

Fig 1 Fuzzy Inference System used to find the next hop selection factor

TABLE II Rules used for getting output NH.

\begin{tabular}{|c|c|c|c|}
\hline E_remaining & Time Factor & Distance & Next hop selection factor \\
\hline Low & Low & Low & Low \\
\hline Low & Low & High & Low \\
\hline Low & High & Low & Low \\
\hline Low & High & High & Low \\
\hline High & Low & Low & High \\
\hline High & Low & High & High \\
\hline High & High & Low & High \\
\hline High & High & High & Low \\
\hline
\end{tabular}

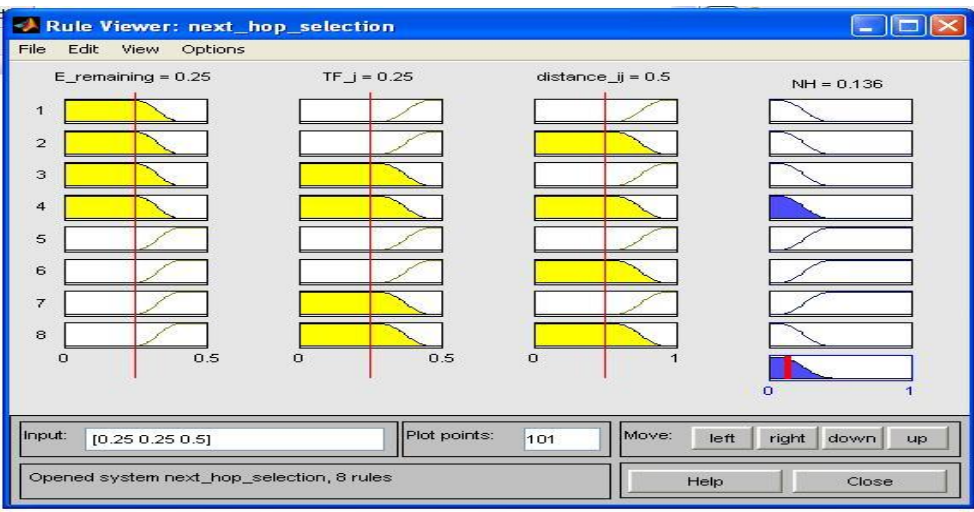

Fig 2 Rule viewer for Next hop selection factor

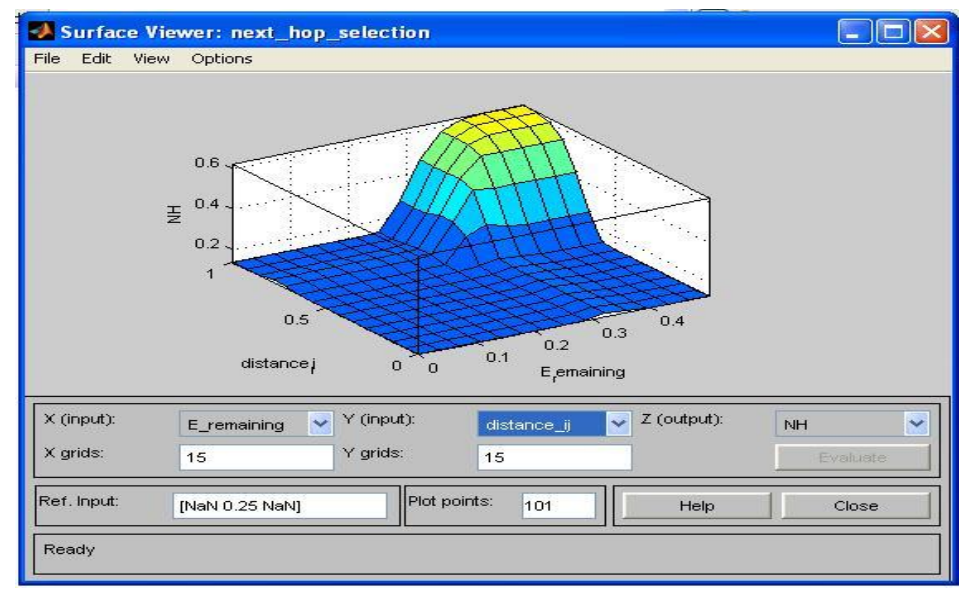

Fig 3 surface view for next hop selection considering Eremaining and distance as inputs 


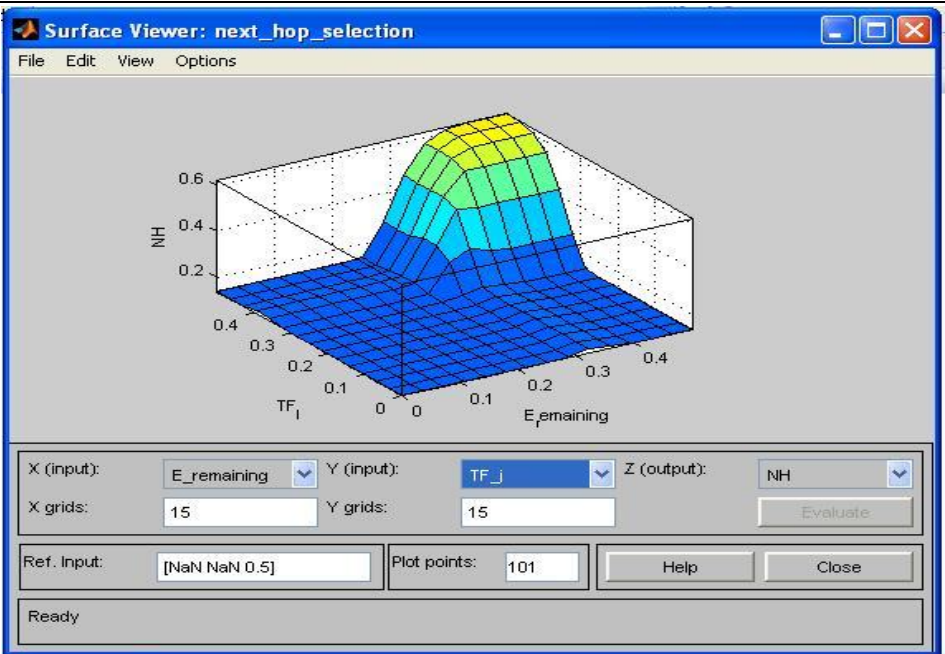

Fig 4 surface view for next hop selection considering Eremaining and Time factor as inputs

\section{Conclusion}

Thus we conclude that in the case of wireless communication the time taken for the packets to be sent and received is the main factor. The remaining energy decreases with increase in the number of days after deployment of the nodes. The Energy deterioration of WSN is still a subject of interest, because of the challenges involved in it. The results show that the node with higher remaining energy, lower time factor and lower distance has more probability of being selected as the next node in forwarding the data to BS. High remaining energy is our main criteria. Among other two parameters TF and distance if any one condition satisfied then the probability for next hop was considered as high.

\section{References}

[1] Meenakshi.B, P.Anandhakumar, “A Novel Energy efficient Surface water Wireless Sensor Network Algorithm”, IndJCSE, ISSN : 0976-5166 Vol. 3 No.3 Jun-Jul 2012 ,pp 494-505

[2] Wendi Rabiner Heinzelman, Anantha Chandrakasan, and Hari Balakrishnan, "Energy-Efficient Communication Protocol for Wireless Microsensor Networks", Proc. Of the $33^{\text {rd }}$ Hawaii International conference on system sciences 2000, IEEE, pp1-10

[3] M. J. Handy, M. Haase, D. Timmermann, "Low Energy Adaptive Clustering Hierarchy with Deterministic Cluster-Head Selection", 2002 IEEE, pp368-372

[4] Linping Wang,Wu Bi,Tingwen Lu,Chi Zhang, “An Improved Energy-efficient Algorithm based on L-DCHS in WSN”, 2010 IEEE, pp312-316

[5] Linping Wang,Wu Bi,Tingwen Lu,Zhen Cai,Zufeng Wang, “A Novel Energy-efficient Algorithm for Wireless Sensor Networks”, 2010 IEEE, Vol.2, pp471-474

[6] "Real-time Wireless Sensor Network for Landslide Detection", Maneesha V. Ramesh, Proc. of the Third International Conference on Sensor Technologies and Applications, 2009, 978-0-7695-3669-9/09 2009 IEEE,DOI 10.1109/SENSORCOMM.2009.67.

[7] K. Premkumar and Anurag Kumar, " Optimal sleep-wake scheduling for quickest intrusion detection using sensor networks", In Proc. IEEE Infocom, Arizona, USA, Apr 2008.

[8] K. Premkumar, Anurag Kumar, and Joy Kuri. Distributed detection and localization of events in large ad hoc wireless sensor networks.In Proc. Forty-Seventh Annual Allerton Conference on Communication, Control,and Computing, 2009.

[9] K. Premkumar, Anurag Kumar, and Venugopal V. Veeravalli. Bayesian quickest transient change detection. In Proc. Fifth International Workshop on Applied Probability (IWAP), 2010.

[10] Jacques Panchard, Jacques Panchard, Prabhakar T. V., Jean-Pierre Hubaux, H. S. Jamadagni, "COMMONSense Net: A Wireless Sensor Network for Resource-Poor Agriculture in the Semiarid Areas of Developing Countries”, Volume 4, Number 1, Fall 2007

[11] Raul Moraisa, Miguel A. Fernandesb, Samuel G. Matosb, Carlos Ser odioa,b, P.J.S.G. Ferreirac, M.J.C.S. Reisa,b, "A ZigBee multi-powered wireless acquisition device for remote sensing applications in precision viticulture", computers and electronics in agriculture 62 ( 2008 ) 94-106, doi:10.1016/j.compag.2007.12.004

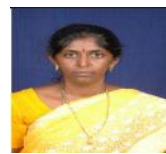

B.Meenakshi received the B.E. degree in Electrical and Electronics Engineering from the Alagappa Chettiar college of Engineering and Technology, Madurai Kamaraj University, India, in 1990, the M.E. degree in Applied Electronics from the Hindustan college of Engineering and Technology, Anna University, Chennai, India in 2005. She is currently pursuing the Ph.D. degree in Information and Communication Engineering, Anna University, Chennai, India. Her research interests include wireless networks, sensor networks, network performance evaluation, and Energy Analysis of wireless networks. She is Assistant Professor of Department of Electrical and Electronics, Sri Sairam Engineering College, Chennai, India.

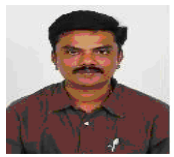

P.Anandhakumar received his B.E degree in Electronics and Communication Engineering from the Government College of Engineering, Salem, India in the year 1994. He obtained his M.E degree in Computer Science and Engineering from Government College of Technology, Coimbatore, India in 1997 and $\mathrm{PhD}$ degree in computer science and Engineering from Anna University, Chennai in 2006.He is the distinguished Professor of Department of Information Technology, Madras Institute of Technology, Chennai, India. His research areas include Mobile computing, Image processing, Robotics, Pervasive computing. His research work has been published in many journal papers and numerous conference papers. 